\title{
PERTINENCIA DE LAS COMPETENCIAS DEMOSTRADAS EN TRABAJOS DE GRADO DE INGENIERÍA FORESTAL
}

\author{
PERTINENCE OF THE COMPETENCIES DEMONSTRATED \\ IN UNDERGRADUATE PROJECTS OF FOREST ENGINEERING
}

\author{
Diana Marcela Niño Pinilla ${ }^{1}$ \\ Angie Tatiana Pérez Reyes ${ }^{2}$ \\ Jaime Alberto Moreno Gutierrez ${ }^{3}$ \\ 1, 2 Ingeniero Forestal, Universidad Distrital Francisco José de Caldas, Bogotá - Colombia. \\ ${ }^{3}$ Ingeniero Forestal; M.Sc. Economía; M.Sc. Administración; Profesor Asociado, \\ Universidad Distrital Francisco José de Caldas, Bogotá - Colombia.

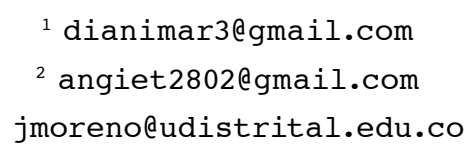

\section{RESUMEN}

Este estudio analiza la pertinencia de las competencias desarrolladas en los trabajos de grado por los estudiantes del proyecto curricular de Ingeniería Forestal en la Universidad Distrital Francisco José de Caldas, con respecto al perfil profesional adoptado y aprobado por el Ministerio de Educación. Para lo anterior, se recopilaron los trabajos de grado sustentados en el periodo 2015 - 2017, desarrollados por los estudiantes formados con el currículo actualizado en 2009 para satisfacer dicho perfil. Los trabajos se clasificaron por competencia, para cada una de las cuales se definieron criterios de evaluación, con base en las características comunes de los temas abarcados por estas, con cuatro niveles de desarrollo de cada competencia, con lo que se procedió a realizar la calificación de los trabajos de grado. Se encontraron un total de 67 trabajos de grado que hacen parte del nuevo pensum, aprobados y sustentados hasta el año 2017 de los cuales se logró tener acceso a 62 para realizar la evaluación. No se evidenció una proporcionalidad entre el desarrollo de las competencias demostradas y la cantidad de trabajos en cada una; por otro lado, se observó que la mayoría de las competencias abarcan muchos temas con lo cual demuestran generalidad, pero pierden especificidad, lo que evidencia en la mayoría de los casos un desarrollo muy básico de las competencias que debe tener un Ingeniero Forestal formado en la Universidad Distrital.

Palabras clave: Desarrollo de competencias, pertinencia del plan de estudios, criterio de evaluación, nivel de desarrollo. 


\section{ABSTRACT}

This study analyzes the relevance of the competences developed in undergraduate projects of the students within the Forest Engineering curriculum project at the Francisco Jose de Caldas District University, with respect to the professional profile adopted and approved by the Ministry of Education; hence, the undergraduate dissertation defenses approved between 2015 and 2017 developed by the students trained with the updated curriculum in 2009 to satisfy this career profile were compiled. The projects were classified by competence; for each evaluation criteria were defined, based on the common characteristics of the subjects covered by these projects, with four levels of development of each competence, which proceeded to make the qualification of the undergraduate projects. A total of 67 undergraduate projects with dissertation defense approved until 2017 were found as part of the new curriculum, whose 62 were granted access to develop the evaluation. There was no evidence of proportionality between the development of the demonstrated competences and the number of projects in each one. On the other hand, it was observed that most of the competences cover many subjects whereby they demonstrate generality but lose specificity, which in most cases evidences a very basic development of the skills of a forest engineer trained in the District University.

Key words: Skills development, curriculum relevance, evaluation criteria, development level.

\section{Resumen Gráfico}

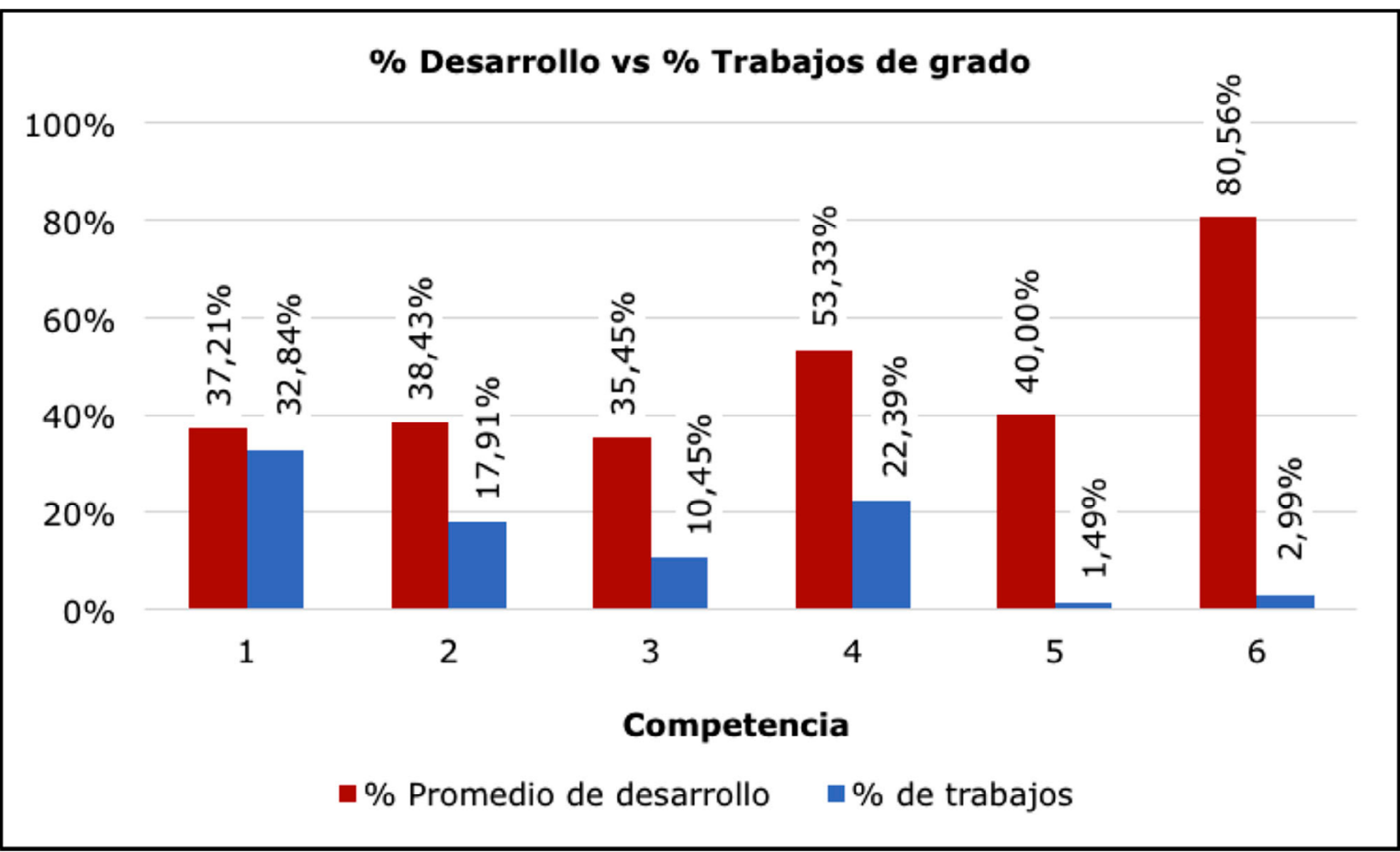




\section{INTRODUCCIÓN}

Según una fuente consultada de Fernow (1993), citada por Shirley (1964) la enseñanza forestal surgió en Alemania en 1763 pero fue hasta 1875 que se incorporaron las escuelas de formación forestal a las universidades iniciando en Austria y ya hasta el siglo XX en América Latina. En Colombia, la enseñanza Forestal llega en 1948 y se consolida en 1952 cuando se crea la Universidad Municipal -hoy Universidad Distrital Francisco José de Caldas (en adelante FJdeC)- y actualmente es enseñada en 6 instituciones educativas del país: Universidad Distrital, Universidad Nacional de Colombia, Universidad del Tolima, Universidad Industrial de Santander, Universidad del Cauca y en el Instituto Tecnológico del Putumayo (Clavijo et al., 2009). La Universidad Distrital es la institución que mayor número de Ingenieros Forestales ha titulado en Colombia -entre 1955 y 2009 aproximadamente 1800(Franco, 2009), con diferentes modalidades de grado, lo cual ha aportado a la investigación forestal en el país.

Vasco (2003) define las competencias como la capacidad de realizar tareas diferentes a las tareas rutinarias que se realizaron mientras se aprendía de un tema específico; es importante tener en cuenta el enfoque de las competencias en la educación superior debido que permite aumentar la pertinencia en los programas, y evaluar la calidad de desempeño y de evaluación de la formación (Tobón, 2008). En Colombia, el Instituto Colombiano para el Fomento de la Educación Superior -ICFES- es el encargado de emitir directrices en evaluación por competencias para la educación superior, donde define dos tipos de competencias: las genéricas, que se refieren a competencias que son generales en una rama profesional $y$, las competencias específicas que son propias de cada profesión (Jiménez,2011 \& ICFES,2018), cada institución educativa define sus competencias específicas de acuerdo con el enfoque que da a la formación de sus estudiantes.

Para agosto de 2005, el proyecto curricular de Ingeniería Forestal de la Universidad Distrital FJdeC logró obtener el reconocimiento de Acreditación de Alta Calidad, vigente hasta el año 2012; posteriormente, por medio del Acuerdo 009 y Resolución 035 expedidos por el Consejo Académico, implementó el Sistema de Créditos Académicos en el año 2006. El proyecto curricular ha instaurado múltiples estrategias que han permitido que sus graduados continúen siendo competitivos dentro del mercado nacional e internacional; parte de estas estrategias han sido la modificación en el plan de estudios basado en competencias, según se vaya requiriendo con el fin de involucrar y adaptarse a las nuevas orientaciones de la profesión, y la búsqueda de la acreditación de alta calidad, para acogerse al sistema de créditos académicos y solucionar las falencias que se evidenciaron con el tiempo hasta llegar al plan actual (Franco, 2017).

Para optar por el título de Ingeniero Forestal es necesario dar una demostración de las competencias adquiridas a lo largo de la carrera universitaria, lo cual se evidencia a través del requisito trabajo de grado, que tiene diferentes modalidades que permiten llevar a la práctica el conocimiento adquirido (Acuerdo 027 de 1993 - expedido por el CSU), por ende, el presente artículo tiene como objetivo analizar la pertinencia de las competencias desarrolladas en los trabajos de grado, por los estudiantes con el perfil vigente planteado por el proyecto curricular de Ingeniería Forestal en la Universidad Distrital Francisco José de Caldas establecido con el cambio de pensum en el año 2009-3. 


\section{MATERIALES Y MÉTODOS}

Para el desarrollo del presente estudio, la metodología se dividió en cuatro fases, cada una compuesta del respectivo conjunto de actividades para su desarrollo, que permitieron dar alcance al objetivo general planteado (Figura 1) adaptando las metodologías propuestas por Aguilera \& Pino (2015), Valderrama et al. (2009), Gairín et al. (2009) y Clavijo (2008).

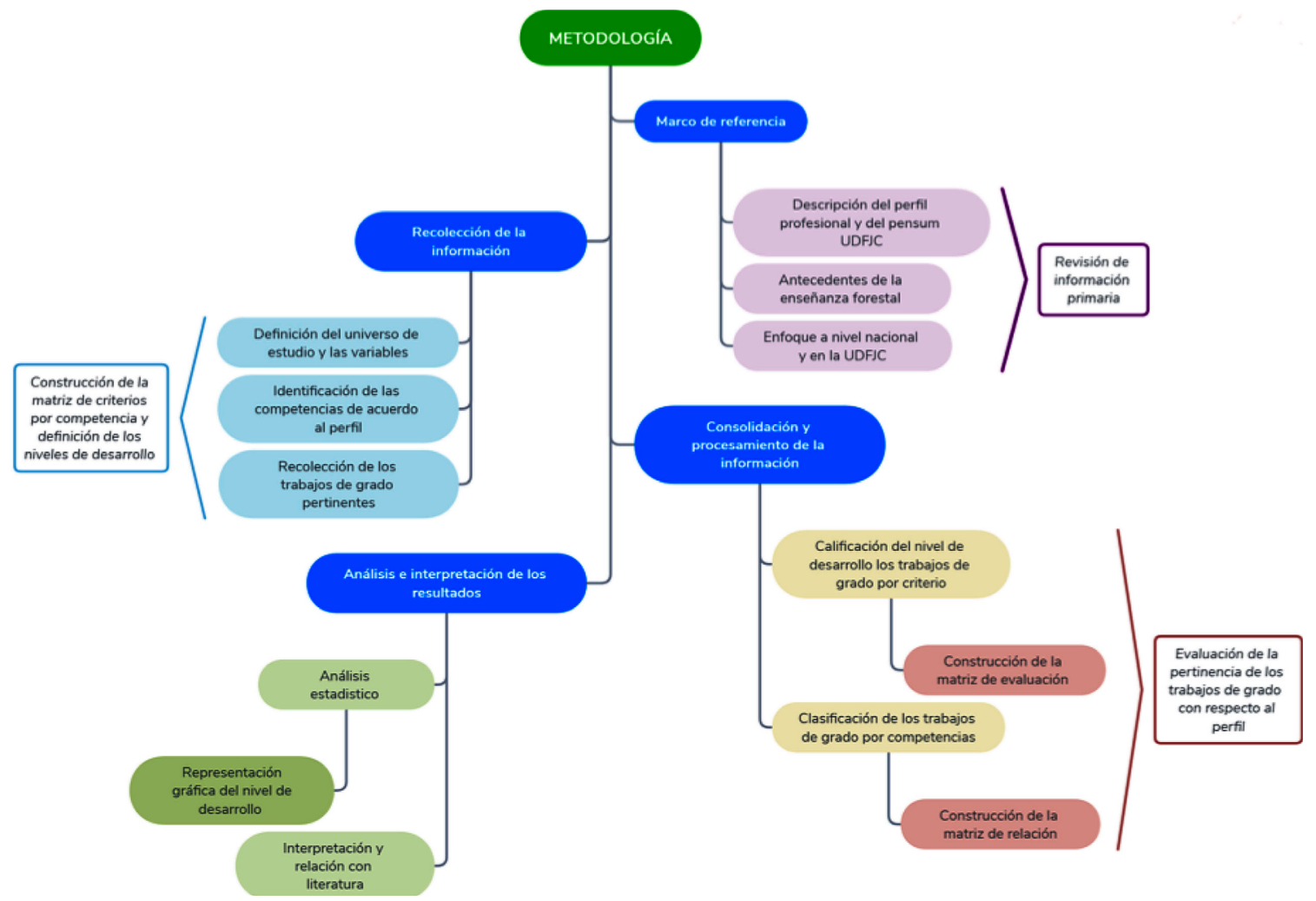

Figura 1. Mapa mental de la metodología propuesta.

Marco de referencia de las competencias del ingeniero forestal Universidad Distrital FJdeC

En esta fase se realizó la consolidación de los antecedentes de la enseñanza forestal tanto en Colombia como a nivel mundial, su influencia e importancia, el enfoque a nivel nacional y en la Universidad y el perfil profesional planteado en el Proyecto Educativo del Programa de Ingeniería Forestal (PEP) de la Universidad Distrital en su última actualización del año 2017. Con esa información, se determinaron los fundamentos y el conocimiento base para el análisis de las competencias específicas definidas para Ing. Forestal en la Distrital en el PEP del año 2008, cómo se demuestran estas competencias con los trabajos de grado, y el reflejo en los trabajos de grado de dichas competencias.

Establecimiento de la base de datos de trabajos de grado realizados bajo el pensum 2009-3

Esta fase se compuso de tres actividades: i) Definición de las variables necesarias para el análisis y recolección de los trabajos de grado (Autor, título, tipo de trabajo de grado, año, 
código y director); ii) Identificación de competencias específicas de acuerdo con el perfil planteado en el PEP 2008; y iii) Recolección de los trabajos de grado pertinentes en el Repositorio Institucional de la Universidad Distrital (RIUD), con los mismos autores y/o directores; este último, hace referencia a los trabajos de grado de los estudiantes que hacen parte del nuevo plan de estudios; es decir, quienes ingresaron a la universidad a partir del periodo 2009 - 3, y sustentaron su trabajo de grado hasta el año 2017.

Se determinó el universo de estudio y los parámetros de evaluación, seleccionando los trabajos de grado sustentados en el periodo comprendido entre el año 2015 y 2017 que hacen parte del nuevo pensum (sistema de créditos). Como variable a manejar se seleccionó el nivel de desarrollo de las competencias demostrado en los trabajos de grado, la cual es de tipo cualitativa (el nivel de desarrollo de las competencias a través de la caracterización de las mismas) y categórica (evaluadas por medio de cuatro niveles). Luego, se definieron criterios de desarrollo para cada competencia, tomando como referencia lo trabajado por Alsina et al. (2013), con cuatro niveles de desarrollo por criterio:

- Nivel de 0: cuando no hay ninguna expresión del criterio en el trabajo de grado (No hay desarrollo)

- Nivel de 1: cuando se desarrollan algunos elementos de los que componen el criterio de evaluación (Desarrollo bajo)

- Nivel de 2: cuando hay un desarrollo de la mayoría de los elementos que componen el criterio (Desarrollo moderado)

- Nivel de 3: cuando hay total desarrollo de los elementos que componen el criterio (Desarrollo perfecto)

Se realizó una interpretación de la definición de las competencias, en la que se evidenciaron los principales componentes para definir los criterios de evaluación teniendo en cuenta que las competencias representan la combinación de conocimiento, actitudes, aptitudes, y destrezas de forma transdisciplinaria, que permitan dar solución y/o respuesta a una necesidad o demanda del medio (García, 2011). Para los niveles 1 a 3 se realizó una matriz de criterios con base en información secundaria, en la que se especificaron los elementos que debían desarrollarse en cada nivel por criterio y por competencia.

\section{Parámetros de identificación de compe- tencias en los trabajos de grado}

Esta fase se compuso de la construcción de la matriz de relación, en la cual se clasificaron los trabajos de grado de acuerdo con las competencias demostradas; posteriormente, se llevó acabo la identificación de las competencias demostradas por los trabajos de grado con las competencias planteadas en el perfil; y finalmente, se determinó la pertinencia de los trabajos de grado, mientras más próxima la calificación a tres, más pertinencia hay.

Para la matriz de relación, los trabajos de grado se clasificaron en cada una de las competencias con base en el objetivo general; subsiguientemente, se realizó la evaluación de cada trabajo de acuerdo con los criterios establecidos para cada competencia, con lo cual se construyó una nueva matriz con el grado de desarrollo demostrado por cada trabajo de grado.

\section{Evaluación del desarrollo de la competen- cia en los trabajos de grado}

Fase final en la cual se realizó el análisis de la información e interpretación de los resultados, a partir de un análisis estadístico de la calificación realizada para determinar la pertinencia de los trabajos de grado en relación con el perfil profesional, según el nivel de desarrollo de la competencia demostrada de acuerdo con los criterios definidos. 
Para lo anterior y con el fin de establecer el nivel de desarrollo demostrado para cada competencia, de acuerdo con Drake, F. (2009) en el libro "La educación forestal en América Latina. Realidad y desafíos para la formación profesional del siglo XXI" se implementó una metodología para la interpretación de la información, en la que se determinó una calificación promedio por criterio y por competencia reflejada en los documentos; con lo cual, se obtuvo el porcentaje promedio de desarrollo demostrado por los trabajos de grado. Por otro lado, se estableció la representatividad de los trabajos de grado por competencia, y el nivel de desarrollo de cada una en los trabajos.

En cuanto al desarrollo general de las competencias, se determinó el nivel promedio de desarrollo por competencias, y se realizó una comparación entre la cantidad de trabajos de grado evaluados (\%) y el promedio de desarrollo demostrado. Finalmente, se determinó la frecuencia de los niveles de desarrollo por cada criterio y por competencia.

\section{RESULTADOS Y DISCUSIÓN}

Para cada una de las competencias se definieron entre tres y nueve criterios de evaluación (Ver anexo Tabla 1) de acuerdo con las características comunes de los temas abarcados por cada una, con base en literatura y los syllabus del plan de estudios.

Se encontraron un total de 67 trabajos de grado aprobados y sustentados hasta el año 2017 que hacen parte del nuevo pensum, de los cuales se logró tener acceso a 62 para realizar la evaluación; al ser clasificados por competencias no fue posible ubicar dos trabajos dentro de las competencias específicas, aun cuando se evidenció un desarrollo de temáticas forestales y de las competencias generales descritas en el PEP, un trabajo de grado no evidencia el desarrollo de ninguna de las temáticas forestales correspondientes a las competencias que alimentan el perfil. De los 59 trabajos de grado restantes, veintidós se evaluaron en la competencia 1 , doce en la competencia 2 , siete en la competencia 3, quince en la competencia 4, uno en la competencia 5 y dos en la competencia 6 (Tabla 2).

Tabla 2. Síntesis del nivel de desarrollo de los trabajos de grado

\begin{tabular}{|c|c|c|c|c|c|c|c|}
\hline Descripción & $\begin{array}{c}\text { Cantidad } \\
\text { T.G. }\end{array}$ & \% de T.G. & $\begin{array}{c}\text { Calificación } \\
\text { promedio }\end{array}$ & $\%$ Pd & Aporte & $\begin{array}{l}\text { Nivel Pd } \\
\text { apx. }\end{array}$ & $\begin{array}{c}\text { Nivel } \\
\text { Pd }\end{array}$ \\
\hline Sin competencia & 2 & $3,23 \%$ & N.A. & N.A. & N.A. & N.A. & N.A. \\
\hline No forestal & 1 & $1,61 \%$ & N.A. & N.A. & N.A. & N.A. & N.A. \\
\hline Competencia 1 & 22 & $35,48 \%$ & 10,05 & $37,21 \%$ & $13,20 \%$ & 1 & 1,12 \\
\hline Competencia 2 & 12 & $19,35 \%$ & 6,92 & $38,43 \%$ & $7,44 \%$ & 1 & 1,15 \\
\hline Competencia 3 & 7 & $11,29 \%$ & 9,57 & $35,45 \%$ & $4,00 \%$ & 1 & 1,37 \\
\hline Competencia 4 & 15 & $24,19 \%$ & 4,80 & $53,33 \%$ & $12,90 \%$ & 2 & 1,60 \\
\hline Competencia 5 & 1 & $1,61 \%$ & 6,00 & $40,00 \%$ & $0,65 \%$ & 1 & 1,20 \\
\hline Competencia 6 & 2 & $3,23 \%$ & 14,50 & $80,56 \%$ & $2,60 \%$ & 2 & 2,42 \\
\hline $\begin{array}{l}\text { Totales / } \\
\text { Promedios }\end{array}$ & 62 & $100 \%$ & 8,64 & $47 \%$ & $6,80 \%$ & 1,33 & 1,48 \\
\hline
\end{tabular}

Dónde: T.G. corresponde a trabajos de grado y Pd a Promedio de desarrollo. 
El nivel promedio de desarrollo es de bajo a moderado, la competencia que se vio más representada fue la 1 con el $35,48 \%$ y un promedio de desarrollo bajo (nivel 1), con un \%Pd del 37,21\%; por otro lado, la competencia 6 se vio poco representada con el $3.23 \%$ de los trabajos de grado, pero tuvo un \%Pd del $80,56 \%$ (Tabla 2). La competencia tres fue la que demostró un desarrollo más bajo con el $35,45 \%$ y fue la tercera con menor representatividad con el 11,29\%; la competencia 1 fue la segunda con desarrollo más bajo con un $\mathrm{Pd}$ de $37,21 \%$. La segunda competencia más representada fue la competencia cuatro con el $24,19 \%$ de los trabajos de grado evaluados, siendo esta también la segunda con mayor porcentaje promedio de desarrollo con el $53,33 \%$.

Con el fin de determinar qué porcentaje representa cada nivel dentro de las competencias y por criterio, se halló la frecuencia relativa y absoluta de cada una y por nivel (Figura 2). Las competencias 2 y 3 evidenciaron un grado de no desarrollo en todos sus criterios, en contraste con las competencias 6 y 4 que mostraron un nivel de desarrollo significativo en sus criterios.

Al evaluar el porcentaje de desarrollo por criterio (Figura 3), las competencias 1 a 4 presentaron un porcentaje de desarrollo inferior al $65 \%$ en todos sus criterios; la competencia 5 tuvo un desarrollo de $100 \%$ en uno de sus criterios y dos de ellos del $0 \%$ y la competencia seis mostró un comportamiento prometedor con un $\% \mathrm{Pd}$ superior al $65 \%$ en todos sus criterios.

Al manejar variables cualitativas, la evaluación de las mismas está sesgada debido a que en situaciones de incertidumbre el razonamiento humano no tiene un comportamiento adecuado y se puede ver influenciado su criterio por las características intrínsecas del individuo como resultado el análisis puede verse parcializado (Batanero, 2001; Moritz, 2004 citados por Salcedo, 2013). Por esto, aun cuando se buscó minimizar el sesgo existente a través de una definición de criterios y niveles basados en la literatura, éste permanece debido a que la selección de los mismos y la evaluación de los trabajos de grado fueron realizados bajo la determinación humana, en donde los conocimientos existentes en el área nos permitieron establecer cuáles eran los criterios que más se adaptaban a las competencias demostrando un desarrollo total de las mismas, sin ser demasiado específicos dada la multiplicidad de temas abarcados por las competencias. La evaluación también se ve sesgada dado que, con base en unos parámetros, los observadores determinaron el nivel correspondiente mediante un consenso.

Según Gutiérrez (2000) y Coll (2007) la especificidad de las competencias juega un papel importante a la hora de promover y evaluar el aprendizaje de los diferentes tipos de conocimientos, y exige a los involucrados el dominio de unos saberes específicos; lo que se ve demostrado en la competencia 6 que fue la que mejor desarrollo mostró, debido a la especificidad de la misma donde todos los criterios establecidos demostraron un desarrollo de moderado a perfecto, obteniendo una calificación superior a dos en todos los criterios, algo similar sucede con la competencia 4 que obtuvo un desarrollo moderado con un nivel promedio de dos. Aunque en las competencias uno, dos, tres y cinco su porcentaje de desarrollo no varía mucho entre sí, la competencia tres se ve afectada en su \%Pd debido a dos trabajos de grado con un desarrollo de bajo a nulo que tienen un promedio de nivel inferior a 0,6 en ambos casos. 
De acuerdo con Hernández et al. (2007) una muestra pequeña, fomenta un mayor error lo que conlleva estimaciones imprecisas, por lo que en el caso de las competencias cinco y seis no se puede determinar la pertinencia con relación a los trabajos de grado debido al tamaño de la muestra, dado que la competencia 5 solo tiene un trabajo de grado que la desarrolla y la competencia 6, dos; sin embargo, la evaluación de estos trabajos permite dar una aproximación al enfoque de desarrollo de cada una, en donde la seis se muestra oportuna con un nivel entre moderado y perfecto, y en la competencia 5 muestra la necesidad de abarcar de forma más amplia las áreas protegidas y los componentes que las configuran.

Coll (2007) establece que, el ser competente en un área implica tener la capacidad de hacer uso de los conocimientos adquiridos en un ámbito específico para dar solución a una exigencia social o individual. Las competencias específicas: son las relativas a los conocimientos técnicos propios de la titulación. Valderrama et al. (2009) definen las competencias específicas como "Las competencias relativas a los conocimientos técnicos propios de la titulación"; con base en lo anterior, se encontró un trabajo de grado que aun cuando desarroIla competencias generales para afrontar y dar solución a una problemática social o individual de la dimensión ambiental, su enfoque no es forestal por lo cual no encaja en las competencias definidas para el perfil profesional.

La formación no puede percibirse únicamente como la adquisición de una serie de conocimientos dentro de un ámbito, sino que se debe contemplar también la capacidad de utilizar ese conocimiento para dar solución a las demandas y problemáticas sociales e individuales (Tiana, 2011); las competencias surgen de la interrelación entre los conocimientos del área, la transmisión del conocimiento y las habilidades genéricas (Argudin, 2015). En el momento de determinar la correlación existente entre los trabajos de grado y las competencias se encontraron dos trabajos cuyo objetivo general no se ajustaba a ninguna competencia; sin embargo, abarcaron claramente una temática forestal y desarrollaron las competencias generales descritas en el $P E P$, integrando los conocimientos del ámbito forestal con las habilidades genéricas y la transmisión del conocimiento.

Según Argudin (2015), la educación por competencias tiene un enfoque sistémico del desarrollo de habilidades y conocimiento, y se puede determinar mediante criterios específicos que permitan la evaluación para establecer la capacidad de construir conocimiento o desempeñar una actividad con un fin específico; con base en lo anterior, se determinó el desarrollo demostrado por los trabajos de grado a partir de los criterios específicos para cada competencia, el cual no superó el $50 \%$ en las competencia 1,2 y 3 . Al relacionar este desarrollo con la cantidad de trabajos de grado evaluados en cada competencia, se observó que no presenta una proporcionalidad, el desarrollo de las competencias no depende de la cantidad de trabajos desarrollados en la misma.

Se pudo evidenciar que algunos trabajos de grado en su objetivo general se ubican dentro de una competencia, pero en el desarrollo del trabajo se ajustan a otra; a diferencia de otros que abarcan más de una competencia, sin desarrollar ninguna en su totalidad, lo cual deja varios criterios por fuera y demuestra que las competencias son demasiado amplias y abarcan varios temas que requieren cierta especificidad (Gutierrez, 2000). De la misma manera, los trabajos de grado se enfocan en un criterio o dos y no demuestran un desarrollo total de 
la competencia, lo cual no permite que haya una manifestación holística en el que se incluyan todas las dimensiones que esta abarca.

\section{CONCLUSIONES}

Se identificaron seis competencias específicas definidas en el Proyecto Educativo del Programa de Ingeniería Forestal, elaboradas en el año 2008 por los docentes de planta del proyecto curricular, las cuales respondían, en su momento, a un plan de estudios diferente al que se implementó en el año 2009 y que se mantiene vigente; sin embargo, estas competencias abarcan muchas temáticas sin llegar a la especificidad, lo cual no permite tener una adecuada evaluación del saber de las competencias demostrado en los trabajos de grado, por ende se hace necesaria la actualización de estas competencias basados en el plan de estudios vigente.

De los sesenta y dos trabajos de grados evaluados, veintidós se clasificaron en la competencia 1 , doce en la competencia 2 , siete en la competencia 3, quince en la competencia 4 , uno en la competencia 5 y dos en la competencia 6; obteniendo un nivel promedio de bajo a moderado; no obstante, los trabajos desarrollados a la luz de las competencias seis y cuatro demostraron una mayor pertinencia, al presentar un mayor grado de desarrollo de los criterios de evaluación y a la especificidad de las competencias; sin embargo, desde una visión general de las competencias el nivel de desarrollo de las mismas es bajo, lo que permite interpretar que la pertinencia también. Esto puede ser resultado de la amplitud de las competencias en las temáticas abarcadas y a que la mayoría de los trabajos de grado se centran en un sólo tema, dejando de lado la interpretación holística del entorno y sus interacciones.
Dentro de los trabajos de grado evaluados, se encontró que en su mayoría no son pertinentes con respecto a las competencias específicas definidas, debido a que en general toman el enfoque de una sola de las asignaturas del plan de estudio y no evalúa su relación con el entorno; esto demuestra que existe una relación y a su vez pertinencia con el plan de estudios, pero dado que las competencias definidas en el PEP no presentan la especificidad suficiente, y la cantidad de temáticas abarcadas por una sola competencia es demasiado amplia, la relación con los trabajos de grado se muestra baja.

De los 62 trabajos de grado evaluados, nueve presentan un nivel de desarrollo de moderado a perfecto, los cuales pueden considerarse como pertinentes, la mayoría de estos corresponden a las competencias cuatro y seis, las cuales son más específicas en las temáticas abarcadas; por otro lado, quince de los trabajos de grado evaluados presentan un nivel de desarrollo menor a uno, lo cual no demuestra una pertinencia mínima con respecto al perfil definido, en su mayoría correspondientes a la competencia uno.

\section{Literatura citada}

Aguilera, L., \& Pino, Y. (2015). Metodología para la evaluación de la pertinencia e impacto de las tesis defendidas en la facultas de derecho de la Universidad de Holguín. Revista de Ciencias Sociales, 131-164.

Alsina, J., Arguila, A., Aróztegui, M., Arroyo, J., Badia, M., Carreras, A., ... Vila, B. (2013). Rubricas para la evaluación de competencias. Barcelona: Octaedro.

Argudin, Y. (2015). Educación basada en competencias. Magistralis. Vol 20, 39-61.

Cantillo, E., Rodríguez, K., \& E., A. (2004). Diversidad y caracterización florística estructural de la vegetación arbórea en la Reserva Forestal Carpatos (Guasca Cundinamarca). Colombia Forestal, 5-21. 
Castaño-Villa, G. (2005). Áreas protegidas, criterios para su selección y problemáticas en su conservación. Boletín Científico - Centro de Museos - Museo de Historia Natural V10, 79-101.

Clavijo, G. (2008). La evaluación del proceso de formación. Cartagena: GACC.

Clavijo, G., Becerra, J., Albarracín, G., \& Silva, L. (2009). Historia y creación de la Universidad Distrital y de la Facultad de Ingeniería Forestal. En ACIF, Historia y Aportes de la Ingeniería Forestal en Colombia, 27 - 36. Bogotá, Colombia.

Coll, C. (2007). Las competencias en la educación escolar: algo más que una moda y mucho menos que un remedio . Aula de Innovación Educativa, 34-39.

Corantioquia. (2013). Plan de ordenación forestal zona de reserva forestal del río Magdalena, bajo Cauca nordeste de Antioquia municipios de Remedios, Segovia, Zaragoza, El Bagre y Nechi.

Crespi, A., Fernandes, C., Castro, A., Bernardos, S., Janiak, A., Pereira, A., ... Santos, C. (2003). Propuesta metodológica para la caracterización estructural de las comunidades vegetales y los grupos funcionales. V17, 345-358.

Drake, F. (2009). La Educación Forestal en América Latina. Realidad y Desafios para la Formación Profesional del Siglo XXI. Santiago, Chile: FAO.

FAO. (2018). FAO Ámbito prioritario. Obtenido de Ordenación Forestal Sostenible: http://www.fao. org/docrep/015/i2763s/I2763S04.pdf

Franco, R. (2009). Egresados Forestales, tan Diversos como Dispersos. Historia y Aportes de la Ingeniería Forestal en Colombia. Bogotá, Colombia: ACIF.

Franco, R. (2017). Consulta sobre el cambio de pensum de Ingeniería Forestal en el año 2009; Universidad Distrital Francisco Josè de Caldas.

Gairín, J., Armengol, C., Gisbert, M., García, M., Rodríguez, D., \& Cela, J. (2009). Guía para la evaluación de competencias en el área de ciencias sociales. Barcelona: Agència per a la Qualitat del Sistema Universitari de Catalunya.

García, J. (2011). Modelo educativo basado en competencias: Importancia y necesidad. Revista electrónica: Actualidades investigativas en educación, 11(3), 1-24.

Gutierrez, A. (2000). Educación basada en competencias: ¿Una alternativa de transformación del curriculum? En La educación para el siglo XXI. Un puente en la cuenca del Pacífico (págs. 143-148). Mexico DF: Biblioteca de la Educación Superior.

Hernández, R., Fernández, C., \& Baptista, P. (2007). Fundamentos de metodología de la investigación. Madrid: McGraw Hill.

ICFES. (2018). ICFES. Obtenido de Glosario: http:// www.icfes.gov.co/atencion-al-ciudadano/glosario

Iturre, M., \& Araujo, P. (2006). Crecimiento y producción del rodal regular. Argentina: UNSE.

Jiménez, C. I. (2011). La formación por competencias: una estrategia integral de educación o un paradigma de la globalización. UTP, 35.

Leython, S., \& Ruiz, T. (2006). Caracterización florística y estructural de un bosque estacional en el sector la Trilla, Parque Nacional Henri Pittier, Estado Aragua, Venezuela. Acta Botánica Venezuelica, 303-314.

López-Camacho, R. (2008). Productos Forestales no Maderables: Importancia e Impacto de su Aprovechamiento. Colombia Forestal 11(1), 215-231.

MADS. (2014). Cartilla orientadora para la gestión del riesgo en incendios forestales en el marco de la Ley 1523 de 2012.

Salcedo, A. (2013). Educación Estadística en América Latina: Tendencias y Perspectivas. Programa de Cooperación Interfacultades. Universidad Central de Venezuela, 361-384.

Shirley, H. (1964). La enseñanaza forestal profesional. Unasylva, 18(4).

Tiana, A. (2011). Análisis de las competencias básicas como núcleo curricular en la educación obligatoria española. Bordón. 63 (1), 63-75.

Tobón, S. (2008). La formación basada en competencias en la educación superior: El enfoque complejo. Universidad Autónoma de Guadalajara, 30.

Valderrama, E., Rullán, M., Sánchez, F., Pons, J., Cores, F., \& Bisbal, J. (2009). La evaluación de competencias en los trabajos fin de estudios. $X V$ Jenui, 405-412.

Vasco, C. (2003). Objetivos específicos, indicadores de logros y competencias ¿y ahora estándares? Educación y cultura, 33-41.

Young, R. (1994). Utilización de los productos forestales: tecnología para un uso inteligente. Revista Internacional de Silvicultura e Industrias Forestales Vol. 45. FAO. 


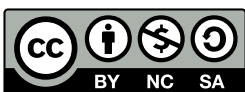

\section{Licencia de Creative Commons}

Revista de Investigación Agraria y Ambiental is licensed under a Creative Commons Reconocimiento-NoComercial-CompartirIgual 4.0 Internacional License. 
